Mon. Not. R. Astron. Soc. 000, 114 (2017) Printed 13 June $2018 \quad$ (MN LATEX style file v2.2)

\title{
A complete disclosure of the hidden type-1 AGN in NGC 1068 thanks to 52 years of broadband polarimetric observation
}

\author{
F. Marin ${ }^{\star}$ \\ Université de Strasbourg, CNRS, Observatoire Astronomique de Strasbourg, UMR 7550, F-67000 Strasbourg, France
}

Accepted 2018 June 8; Received 2018 June 7; in original form 2018 April 12

\begin{abstract}
We create the first broadband polarization spectrum of an active galactic nucleus (AGN) by compiling the $0.1-100 \mu \mathrm{m}, 4.9 \mathrm{GHz}$ and $15 \mathrm{GHz}$ continuum polarization of NGC 1068 from more than 50 years of observations. Despite the diversity of instruments and apertures, the observed spectrum of linear continuum polarization has distinctive wavelength-dependent signatures that can be related to the AGN and host galaxy physics. The impact of the Big Blue bump and infrared bump, together with electron, Mie scattering, dichroism and synchrotron emission are naturally highlighted in polarization, allowing us to reveal the type- 1 AGN core inside this type- 2 object with unprecedented precision. In order to isolate the AGN component, we reconstruct the spectral energy distribution of NGC 1068 and estimate the fraction of diluting light in the observed continuum flux. This allows us to clearly and independently show that, in the case of NGC 1068, Thomson scattering is the dominant mechanism for the polarization in the optical band. We also investigate the effect of aperture on the observed polarization and confirm previous findings on the extension of the narrow line region of NGC 1068 and on the B-band and K-band polarization from the host. Finally, we do not detect statistically significant aperture-corrected polarimetric variations over the last 52 years, suggesting that the parsec-scale morphological and magnetic geometries probably remained stable for more than half a century.
\end{abstract}

Key words: catalogues - galaxies: active - galaxies: fundamental parameters galaxies: nuclei - galaxies: Seyfert - polarization

\section{INTRODUCTION}

NGC 1068, also commonly known as M77, is one of the most studied active galactic nuclei (AGN). Its proximity to Earth $\left(z=0.003793, d^{1}=10.582_{-3.402}^{+5.418} \mathrm{Mpc}\right)$ and its bolometric luminosity $\left(\sim 2 \times 10^{45} \mathrm{erg} \mathrm{s}^{-1}\right.$, Hönig et al. 2008, Raban et al. 2009) make NGC 1068 an ideal target for spectroscopic, polarimetric and high resolution angular imaging studies. It allows high signal-to-noise ratio observations (e.g., Low \& Rieke 1971; Wilson \& Elvis 1997, Alexander et al. 1999 Bauer et al. 2015), which is imperative to distinguish the central AGN from its host spiral galaxy Balick \& Heckman 1985). It is an essential point as the properties of the intermediate region between the inner AGN dust material and the outer circumnuclear starforming regions of NGC 1068 can probe in great details the co-evolution of the AGN and

\footnotetext{
* E-mail: frederic.marin@astro.unistra.fr

1 Redshift-independent distance computed by the NASA/IPAC

Extragalactic Database from 11 distances in the literature.
}

its host galaxy (Vollmer et al. 2008, Schartmann et al. 2009, 2010).

The AGN in NGC 1068 was the key-point of the Unified Model for radio-quiet objects ${ }^{2}$ such as postulated by Antonucci (1993). This zeroth order geometrical scheme has proven to be particularly successful in explaining a large fraction of observational features in many radio-quiet AGN thanks to a critical point: the nuclear orientation of the central engine. Depending on the inclination of the observer with respect to the polar axis of the object, defined as the direction towards which the outflows of the AGN are directed, a variety of emission lines can be detected. Khachikian \& Weedman (1974) defined two types of Seyferts galaxies. In

\footnotetext{
2 We acknowledge that the spectropolarimetric study of the radio galaxy 3C 234 preceded the work on NGC 1068, revealing a type1 spectrum in polarized light together with a high polarization degree oriented perpendicular to the radio axis (Antonucci 1982, 1984). However 3C 234 is a radio-loud AGN, while NGC 1068 is radio-quiet.
} 
Marin F.

their nomenclature Seyfert-2s show narrow permitted and forbidden emission lines while Seyfert-1s show broad permitted emission lines in addition to the Seyfert-2s lines. The reason for the disappearance of the broad permitted emission lines in types-2s was a crucial question that was solved largely thanks to observations of NGC 1068. Antonucci \& Miller (1985) have shown that high-resolution $(5-10 \AA)$, high signal-to-noise ratio polarization spectra of the nucleus of NGC 1068 are necessary to uncover broad Balmer lines and Fe II emission. Those lines have been found to have an intrinsic polarization $>15 \%$ at approximately the same position angle as that of the continuum, and their line wings appear broadened in the polarized flux spectra only. It was a definite set of proofs that a Seyfert-1 nucleus is hiding inside NGC 1068. The polarization angle, perpendicular to the axis of radio emission, is the strongest evidence for polar scattering of inner AGN photons, imprinting the polarized spectrum with the broad emission line. To explain the absence of broad lines in total flux and the orientation of the polarization angle, the most convenient way is to postulate the existence of a reservoir of circumnuclear dust that is enshrouding the nucleus along the equatorial plane. According to the inclination of the observer the central engine can be directly observed (Seyfert-1s) or it is obscured by dust (Seyfert-2s). It naturally explains the disappearance of the broad permitted emission line that can only be detected in polarized flux. The theory was also confirmed in radio-loud AGN (Antonucci 1984, Barthel 1989), leading to a unification of radio-loud AGN which includes radio galaxies, quasars, and blazars (Urry \& Padovani 1995).

Polarimetric observations of NGC 1068 had a significant impact on our global comprehension of AGN but many questions that can be solved with polarimetry remain open. The systematic search for hidden type-1 cores in Seyfert-2s allows one to test whether all AGN are fundamentally the same in terms of physical components. Optical spectropolarimetry revealed broad components of Balmer lines in many type$2 \mathrm{~s}$ but a fraction of them are reluctant to show broad permitted emission lines in their linearly polarized spectrum (see, e.g., Young et al. 1995; Young 2000, Moran et al. 2000

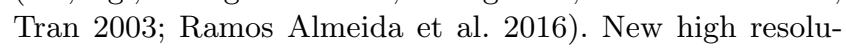
tion, high signal-to-noise ratio polarimetric observations of AGN on modern telescopes with large mirrors are necessary to test if some type- 2 AGN genuinely lack a type- 1 core. In addition, high angular resolution, high contrast polarimetric imaging using adaptive optics have shown that nearby bright AGN can be observed at phenomenal resolutions in the nearinfrared (e.g., Gratadour et al. 2015, Lopez-Rodriguez et al. 2015). Gratadour et al. (2015) used the extreme adaptive optics system on the SPHERE instrument at the Very Large Telescope to observe NGC 1068 in the H $(1.65 \mu \mathrm{m})$ and K' $(2.2 \mu \mathrm{m})$ bands, and achieved polarimetric images with resolution 0.068 " ( $\sim 4 \mathrm{pc}$ at $d \approx 14 \mathrm{Mpc}$, which is the distance usually chosen for this object). By doing so, the authors revealed a compact elongated ${ }^{3}(20 \times 60 \mathrm{pc})$ structure, tracing

3 Atacama Large Millimeter/submillimeter Array (ALMA) observations have revealed that the torus has an extension of $\sim 5 \mathrm{x} 10 \mathrm{pc}$ at $432 \mu \mathrm{m}$ (García-Burillo et al. 2016 Gallimore et al. 2016. Imanishi et al. 2018). The structure suggested by Gratadour et al. (2015) should be taken as an upper limit due to the methodology used by the authors. the scattering regions at the center of NGC 1068, together with the already seen hourglass-shaped polar winds (see, e.g., Capetti et al. 1995a b Kishimoto 1999). The later author pushed the polarimetric study of the ultraviolet linear polarization of the polar outflows up to the determination of the location of the nucleus of NGC 1068 and figured out the three-dimensional structure of its winds.

There is a lot more to be discovered by polarimetry. One can test the geometry of the region responsible for the emission of the broad permitted line (Smith et al. 2002). It should be possible to determine the spin, mass and inclination of the central supermassive black hole thanks to X-ray polarimetry (Dovčiak et al. 2004, Schnittman \& Krolik 2009, 2010, Dovčiak et al. 2011). The composition and location of almost all the AGN components can be probed independently of the fact that they are obscured by dust or gas. Even the presence of aborted jets may be detected. To reach this goal, broadband polarimetry is needed. Ramos Almeida et al. (2016) have clearly showed that information can be missed if we focus on a too narrow waveband. But is there enough published data to test the Unified Model of AGN at all wavebands? Do we need further instruments on large class telescopes? What is the broadband continuum polarization spectrum of radio-quiet AGN and what are the wavebands to be still explored?

To answer those questions, we present a thorough compilation of all the continuum polarimetric observations of NGC 1068 in order to create, for the first time, a combined spectrum of the linear polarization of an archetypal type- 2 AGN. We use the fact that no other AGN have as much published polarimetric data as NGC 1068 to estimate the impact of several observational constraints on the detected polarization levels. We also investigate the time-evolution of polarization over $\sim 52$ years and check whether NGC 1068 has evolved in terms of geometry. By doing so we aim at making a strong case for future polarimetric instruments and programs. In this paper, we compile the published polarimetric data on NGC 1068 in Sect. 2.1 and build the combined polarized spectrum of NGC 1068 in Sect. 2.2. We investigate the effect of apertures in Sect. 2.3 before computing the global spectral energy distribution (SED) of the system in Sect. 2.4. We use this multi-component SED to correct the continuum polarization from starlight dilution in Sect. 2.5 and demonstrate the predominance of electron scattering. Finally, we examine the temporal evolution of the linear polarization of NGC 1068 in Section. 2.6 We discuss our results and the important observations to be made in the future in Sect. 3 before concluding our paper in Sect. 4

\section{THE DATA}

To gather the polarimetric observations published in refereed papers, the SAO/NASA Astrophysics Data System (ADS) was extensively used. The SAO/NASA ADS is a Digital Library portal for researchers in Astronomy and Physics, operated by the Smithsonian Astrophysical Observatory (SAO) under a NASA grant; its website is accessible here: http://adsabs.harvard.edu/. Keywords and filtering by object name (NGC 1068 and its alternative names) allowed for easier detection of relevant papers. Discussions with experts in the field, acknowledged at the end of this 
paper, also helped to find obscure publications. Several journal were also contacted to retrieve non-indexed results mentioned in several publications (Astronomical Journal, Astronomical Circular).

\subsection{The catalog}

The final catalog of polarimetri4 $4^{4}$ observations of NGC 1068 is presented in Tab. 1 It contains 34 publications spanning over more than 50 years $(1965$ - 2017, observational dates, not paper publication time-stamp). All papers are accounting for instrumental polarization, together with interstellar polarization. Due to the high Galactic latitude of NGC $1068\left(-51.93^{\circ}\right)$, Galactic contamination by diffuse interstellar grains is not expected to impact the measured polarization in the ultraviolet - far-infrared band (Prunet et al. 1998). However a large fraction of authors did not corrected their data for dilution/contamination of the polarization by the host galaxy (exceptions include, but are not limited to, the work by Miller \& Antonucci 1983, Antonucci \& Miller 1985, Kishimoto 1999 and Lopez-Rodriguez et al. 2015). The dust lane and host galaxy have a visual extinction $A_{V} \sim 9$ mag to the core of NGC 1068, producing an aperture-dependent level of dilution to be corrected at ultraviolet, optical and infrared wavelengths. Unfortunatelly this correction was not achieved by all the authors. To have a consistent set of measurements, the polarized values extracted from the aforementioned papers are the one that were not corrected for starlight dilution (but see Sect 2.4 for the correction of polarization by removing the host component). The measurements were taken with a variety of instruments, listed in the second column of Tab. 1 Observations were made in different wavebands, from the ultraviolet using the Wisconsin Ultraviolet Photo Polarimeter Experiment (Code et al. 1993) to the far-infrared using the SOFIA High-resolution Airborne Wideband Cameraplus (HAWC+, Lopez-Rodriguez, E., private communication). Only two polarimetric observations have been published in the radio band, an upper limit at $15 \mathrm{GHz}$ (Wilson \& Ulvestad 1982) and a debated measurement at $4.9 \mathrm{GHz}$ (Wilson \& Ulvestad 1983). The bulk of observations were taken in the optical and near-infrared bands since they remain the easiest bands for ground observations. A variety of slits and circular apertures were used, depending on the technology available at that time (fourth column of Tab. 1). Finally, we have identified observations that were achieved in imaging modes. For these cases the authors had polarization maps and could have, in principle, varied the aperture to remove the contribution of the host galaxy.

In Fig. 1, we present the temporal distribution of the number of polarimetric observations listed in our catalog. The very first polarimetric observation of NGC 1068 listed in our sample goes back to 1965 - 1966 (Dibai \& Shakhovskoi 1966). A year later, Dombrovskii \& Gagen-Torn (1968)

\footnotetext{
${ }^{4}$ We focus on NGC 1068 linear polarization. Circular polarization, at least in the near-ultraviolet, optical and near-infrared bands, has been the subject of an unsolved debate. One paper claimed a non-detection (Landstreet \& Angel 1972), another a non-significant detection (Gehrels 1972) and two others a clear detection (Nikulin et al. 1971|| Angel et al. 1976).
}

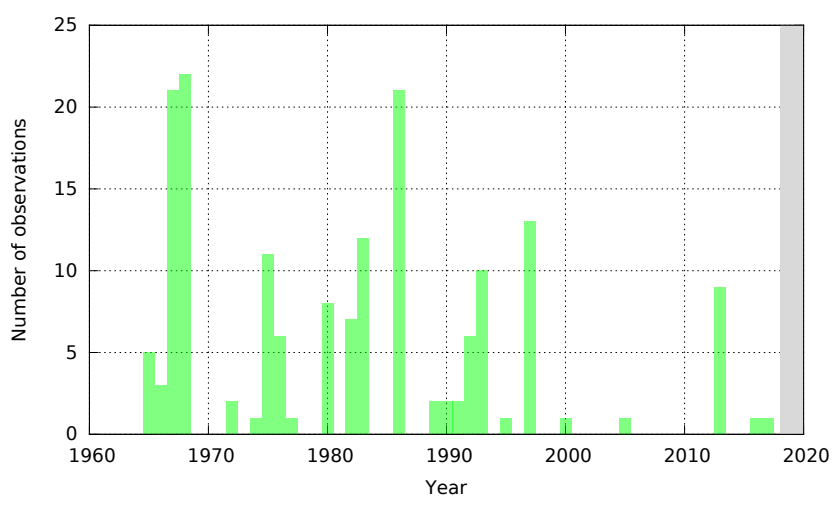

Figure 1. Number of observations per year dedicated to measure the broadband continuum polarization of NGC 1068. The shaded area corresponds to the forthcoming years $2019-2020$.

quoted polarimetric observations "with a somewhat smaller aperture than ours" that might also have been achieved by Merle F. Walker in 1964 but the references listed in Dombrovskii \& Gagen-Torn (1968) point towards papers that are either about other galaxies (M33, Walker 1964) or do not exist at all. A similar reference to the work by Walker (Astron.J., 1964, 69, 682) is mentioned in Elvius \& Hall (1965) but the related paper could not be found. Hence, while we acknowledge that earlier polarimetric observations might have been achieved, we start our catalog in 1965 1966 due to the lack of open-access publications.

The interest of the community for the polarimetric signature of NGC 1068 and similar galactic nuclei grew fast, with more than 50 observations achieved before 1970. The goal was to explore the origin of the observed polarization. Synchrotron emission was one of the two mechanisms (together with scattering) suggested to explain the high ultraviolet polarization in its nucleus (Elvius \& Hall 1965). A few additional polarimetric measurements of this AGN occurred until the advent of the 3.9-m Anglo-Australian Telescope and the Lick 3-m Shane Telescope, which gave a new kick to observations in 1983 thanks to their large mirrors and up-to-date polarimeters. The true scattered polarization of NGC 1068 was estimated to be much higher than previously thought thanks to the careful removal of starlight from the host galaxy by Miller \& Antonucci (1983). In addition, it was found that the position angle of the optical continuum radiation is perpendicular to the axis of radio emission $\left(18^{\circ} \pm 5^{\circ}\right.$, Wilson \& Ulvestad 1982). After the breakthrough achieved in 1985, where Antonucci \& Miller (1985) proved that a Seyfert-1 nucleus lies hidden in the core of NGC 1068, and that the origin of the continuum and broad-line polarization is due to scattering, the number of observations decreased. It was only in the mid of the 90 's, when the Unified Model of AGN was synergized, confirmed and then reviewed (Antonucci 1993), that the community acquired a few more polarimetric observations of NGC 1068. Since the new millennium, only a dozen of polarimetric observations of this AGN have been achieved on 10-m class telescopes. Another observational gap could appear between the era of 10-m and 30-m class telescopes, at least partly driven by the relative lack of polarimeters on such large telescopes. 


\begin{tabular}{|c|c|c|c|c|}
\hline Reference & Instrument & Waveband & Aperture & Imaging \\
\hline Dibai \& $\quad$ Shakhovskoi & integrating electropolarimeter of the & $\mathrm{U}, \mathrm{B}, \mathrm{V}$ filters & $15 ", 25 ”$ & \\
\hline$(1966)$ & Crimean Astrophysical Observatory & & & \\
\hline $\begin{array}{l}\text { Dombrovskii \& Gagen- } \\
\text { Torn }(1968)\end{array}$ & $\begin{array}{l}\text { electropolarimeter of Leningrad Univer- } \\
\text { sity }\end{array}$ & $\begin{array}{l}\mathrm{U}, \mathrm{B}, \mathrm{V}, \mathrm{R} \text { system, ZhS-18 } \\
\text { and SZS-22 filters }\end{array}$ & $13 ", 26 "$ & \\
\hline Kruszewski $(1968)$ & Catalina 154-cm Telescope & $\begin{array}{ll}\mathrm{U}\left(2.78 \mu \mathrm{m}^{-1}\right), & \mathrm{H}(2.33), \\
\mathrm{G}(1.93), \mathrm{O}(1.56), & \mathrm{R}(1.21), \\
\text { and } \mathrm{I}(1.06) & \end{array}$ & $9.2 ", 10.2 "$ & \\
\hline $\begin{array}{l}\text { Visvanathan \& } \quad \text { Oke } \\
(1968)\end{array}$ & $\begin{array}{l}\text { 60- and 100-inch telescopes at Mount } \\
\text { Wilson }\end{array}$ & $\mathrm{U}, \mathrm{B}, \mathrm{V}, \mathrm{R}$ system & $12.5 ", 19.0 ", 28.0 "$ & \\
\hline Kruszewski (1971) & Catalina 154-cm Telescope & $\begin{array}{ll}\mathrm{U}\left(2.78 \mu \mathrm{m}^{-1}\right), & \mathrm{H}(2.33), \\
\mathrm{G}(1.93), \mathrm{O}(1.56), & \mathrm{R}(1.21), \\
\text { and } \mathrm{I}(1.06) & \end{array}$ & 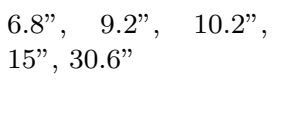 & \\
\hline Knacke \& Capps (1974) & $\begin{array}{l}\text { 1.3-m telescope of the Kitt Peak National } \\
\text { Observatory }\end{array}$ & $\begin{array}{l}3.5 \mu \mathrm{m} \quad(\Delta \lambda=0.6 \mu \mathrm{m}) \\
10.2 \mu \mathrm{m} \quad(\Delta \lambda=6 \mu \mathrm{m}), \quad \text { and } \\
18.4 \mu \mathrm{m}(\Delta \lambda=1 \mu \mathrm{m})\end{array}$ & $12 "$ & \\
\hline Angel et al. 1976 & $\begin{array}{l}90 \text { inch }(2.3 \mathrm{~m}) \text { telescope of Steward Ob- } \\
\text { servatory }\end{array}$ & $3200-8600 \AA$ & $2 "$ & \\
\hline Dyck \& Jones (1976) & unknown & $1.2 \mu \mathrm{m}, 2.2 \mu \mathrm{m}$ & $5.4 ", 11 "$ & \\
\hline Elvius $(1978)$ & $\begin{array}{l}\text { 72-inch Perkins Telescope of the Ohio } \\
\text { Wesleyan Observatory }\end{array}$ & Schott UG1 filter & 13"-long slit & \\
\hline Lebofsky et al. (1978) & Steward Observatory 90-inch telescope & $\mathrm{J}, \mathrm{H}, \mathrm{K}, \mathrm{L}$ ' filters & $4 ", 6 ", 8 "$ & \\
\hline $\begin{array}{lll}\text { Wilson } & \& & \text { Ulvestad } \\
(1982) & & \\
\end{array}$ & Very Large Array & $15 \mathrm{GHz}$ & $0.7 "$ & $\checkmark$ \\
\hline Martin et al. 1983$)$ & $\begin{array}{l}\text { Steward Observatory } 2.3 \mathrm{~m} \text {, Las Cam- } \\
\text { panas } 1.0 \text { and } 2.5 \mathrm{~m} \text {, Kitt Peak } 1.3 \text { and } \\
2.1 \mathrm{~m} \text {, and University of Western Ontario } \\
1.2 \mathrm{~m} \text { telescopes }\end{array}$ & C500 filter (blue - green) & $5 "$ & \\
\hline McLean et al. 1983 ) & 3.9-m Anglo-Australian Telescope & $3900-8000 \AA$ & $1.7 " \times 2.2 ", 2 " \times 2.5 "$ & \\
\hline $\begin{array}{lll}\text { Miller } & \& & \text { Antonucci } \\
(1983) & & \end{array}$ & Lick 3-m Shane Telescope & $3500-5300 \AA$ & $2.8 "$ & \\
\hline \begin{tabular}{|lll} 
Wilson & $\&$ & Ulvestad \\
$(1983)$ & & \\
\end{tabular} & Very Large Array & $4.9 \mathrm{GHz}$ & $1 "$ & $\checkmark$ \\
\hline Aitken et al. $(1984)$ & $\begin{array}{l}\text { 3.9-m Anglo-Australian Telescope and } \\
\text { 3.0-m Infrared Telescope in Hawaii }\end{array}$ & $8-13.1 \mu \mathrm{m}$ & $4.2 ", 5.6 ", 15 ", 50 "$ & \\
\hline $\begin{array}{lll}\text { Antonucci } \quad \& \quad \text { Miller } \\
(1985) & & \end{array}$ & $\begin{array}{l}\text { Lick 3-m Shane Telescope Image Dissec- } \\
\text { tor Scanner }\end{array}$ & $3500-7000 \AA$ & $2.8 "$ & \\
\hline Bailey et al. $(1988)$ & 3.9-m Anglo-Australian Telescope & $0.36-4.8 \mu \mathrm{m}$ & $4.5 ”, 6.0 "$ & \\
\hline Scarrott et al. (1991) & 3.9-m Anglo-Australian Telescope & $\mathrm{V}, \mathrm{K}$ filters & $1.6 ", 2.8 ", 4.5 "$ & $\checkmark$ \\
\hline Code et al. $(1993)$ & $\begin{array}{l}\text { Wisconsin Ultraviolet Photo Polarimeter } \\
\text { Experiment }\end{array}$ & $1500-3200 \AA$ & $6 " \mathrm{x} 12 "$ & \\
\hline Antonucci et al. (1994) & $\begin{array}{l}\text { Hubble Space Telescope Faint Object } \\
\text { Spectrograph }\end{array}$ & $1575-3300 \AA$ & $0.3 ", 1 ", 4.3 \times 1.4 "$ & \\
\hline Capetti et al. 1995a) & $\begin{array}{l}\text { Hubble Space Telescope Faint Object } \\
\text { Camera and Wide Field Planetary Cam- } \\
\text { era }\end{array}$ & $\begin{array}{l}2700-3700 \AA, \quad 5000 \\
6000 \AA\end{array}$ & $2.8 "$ & $\checkmark$ \\
\hline Capetti et al. (1995b) & $\begin{array}{l}\text { Hubble Space Telescope COSTAR- } \\
\text { corrected Faint Object Camera }\end{array}$ & $2400-2700 \AA$ & $2.8 "$ & $\checkmark$ \\
\hline Tran (1995) & Lick 3-m Shane Telescope & $\begin{array}{l}4560-7355 \AA, \quad 4600- \\
7400 \AA, 3315-4400 \AA\end{array}$ & $2.4 "$-slit & \\
\hline Young et al. $(1995)$ & $\begin{array}{l}3.8 \mathrm{~m} \text { United Kingdom Infrared Tele- } \\
\text { scope and the CGS4 spectrometer }\end{array}$ & $\begin{array}{l}0.46-\quad 0.77 \mu \mathrm{m}, \quad 1.18- \\
1.38 \mu \mathrm{m} \text { and } 1.66-2.07 \mu \mathrm{m}\end{array}$ & $3.08 \times 3.0 "$ & \\
\hline Packham et al. (1997) & $\begin{array}{l}\text { IR imaging polarimeter at the Anglo- } \\
\text { Australian Telescope }\end{array}$ & $\mathrm{J}, \mathrm{H}, \mathrm{K}_{\mathrm{n}}$ filters & $2.0 ", 4.5 ", 6.0 "$ & $\checkmark$ \\
\hline Alexander et al. (1999) & $\begin{array}{l}\text { United Kingdom Infrared Telescope, } \\
\text { CGS4 spectrometer and IRPOL2 }\end{array}$ & $1.05-1.35 \mu \mathrm{m}$ & $1.23 " \times 6.7 "$ & \\
\hline Lumsden et al. (1999) & 3.9-m Anglo-Australian Telescope & $\mathrm{J}, \mathrm{H}, \mathrm{K}_{\mathrm{n}}, \mathrm{N}$ filters & $2.0 ", 4.5 ", 6.0 "$ & $\checkmark$ \\
\hline Simpson et al. 2002$)$ & $\begin{array}{l}\text { NICMOS Camera } 2 \text { on the Hubble Space } \\
\text { Telescope }\end{array}$ & $2 \mu \mathrm{m}$ & $3.0 "$ & $\checkmark$ \\
\hline Watanabe et al. (2003) & $\begin{array}{l}3.8 \mathrm{~m} \text { United Kingdom Infrared Tele- } \\
\text { scope }\end{array}$ & $\begin{array}{l}0.46-\quad 0.90 \mu \mathrm{m}, \quad 0.92- \\
1.80 \mu \mathrm{m} \text { and } 1.88-2.50 \mu \mathrm{m}\end{array}$ & $3.5 "$ & \\
\hline Packham et al. (2007) & Gemini North 8.1-m telescope & $9.7 \mu \mathrm{m}$ & $1.7 " \times 1.2 "$ & $\checkmark$ \\
\hline
\end{tabular}

Table 1. Catalog of published polarimetric measurements of NGC 1068. The first column is the reference paper, the second column is the instrument used for the measurement, the third column is the waveband or filters used during the observation, the fourth column is the observation aperture (in arcseconds), and the fifth column indicates if polarimetric images were taken. 


\begin{tabular}{|c|c|c|c|c|}
\hline Reference & Instrument & Waveband & Aperture & Imaging \\
\hline Mason et al. 2007) & $\begin{array}{l}\text { IRPOL2 spectropolarimetry module and } \\
\text { CGS4 on the } 3.8 \mathrm{~m} \text { UK Infrared Telescope }\end{array}$ & $3.10-3.67 \mu \mathrm{m}$ & $0.6 "$ "slit & \\
\hline $\begin{array}{l}\text { Lopez-Rodriguez et al. } \\
(2015)\end{array}$ & MMT-Pol on the $6.5-\mathrm{m}$ MMT & J', K’ filters & $0.2 ", 0.5 ", 2.0 "$ & $\checkmark$ \\
\hline $\begin{array}{l}\text { Lopez-Rodriguez et al. } \\
(2016)\end{array}$ & $\begin{array}{l}\text { CanariCam on the } 10.4-\mathrm{m} \text { Gran Telesco- } \\
\text { pio CANARIAS }\end{array}$ & $\begin{array}{l}8.7 \mu \mathrm{m}, \quad 10.3 \mu \mathrm{m}, \quad 11.3 \mu \mathrm{m}, \\
11.6 \mu \mathrm{m}\end{array}$ & $0.4 ", 2.0 "$ & $\checkmark$ \\
\hline Grosset et al. (in prep.) & SPHERE/VLT & $\mathrm{H}$ and $\mathrm{K}^{\prime}$ bands & $\begin{array}{l}0.2 ", 0.5 ", 1.0 ", 2.0 " \\
3.0 ", 4.0 ", 5.0 "\end{array}$ & $\checkmark$ \\
\hline $\begin{array}{l}\text { Lopez-Rodriguez et al. } \\
\text { (in prep.) }\end{array}$ & HAWC+ on the 2.5-m SOFIA telescope & $53 \mu \mathrm{m}, 89 \mu \mathrm{m}$ & $5.0 ", 8.0 "$ & $\checkmark$ \\
\hline
\end{tabular}

Table 2. Tab. 1 continued.

\subsection{Broadband continuum polarization of NGC 1068}

We compiled all the linear continuum polarization data of NGC 1068 in Fig. 2 When continuum polarization measurements were not estimated by the authors we used WebPlotDigitizer to synthesize the polarization spectrum and extract the relevant numbers. WebPlotDigitizer (https:// automeris.io/WebPlotDigitizer/) is a polyvalent and free software developed to facilitate easy and accurate data extraction from spectra and it has already been used in Marin et al. (2017) to reconstruct the ultraviolet spectropolarimetric spectrum of NGC 1068.

Our final spectrum spans from almost $0.1 \mu \mathrm{m}$ to $100 \mu \mathrm{m}$, together with two additional measurements at $4.9 \mathrm{GHz}$ and $15 \mathrm{GHz}$, with a variety of apertures, signalto-noise ratios and spectral resolutions. In this figure, we did not correct the polarization levels for the presence of diluting starlight emission originating from the host galaxy that may have a significant effect depending of the aperture of the observation/slit. This will be investigated in further details in Sect. 2.3. We plotted the continuum linear polarization in log scale to better contrast the fractional contribution of unpolarized light. The polarized flux is extracted from publications and is compared to a normal type- 1 AGN total flux spectrum to investigate how the cross section of the scatterer is changing with wavelength. The type- 1 template we use was compiled by Prieto et al. (2010) and is the averaged SED of the high spatial resolution SEDs of NGC 3783, NGC 1566 and NGC 7469. The template was rescaled in order to be easily comparable to the polarized flux of NGC 1068. Finally, the polarization position angle has been subtracted from the parsec-scale radio position angle to check whether the polarization angle is parallel or perpendicular to the axis of the radio source associated with the galaxy (Antonucci 1993). We use the parsec-scale radio position angle estimated by Wilson \& Ulvestad (1982) but we acknowledge the fact that the position angle is almost $0^{\circ}$ at sub-arcesond scales (Muxlow et al. 1996). Our choice to use the parsec-scale value is coherent as the bulk of published polarimetric data having apertures larger than 1".

We can see from Fig. 2 that the compiled polarization spectrum of NGC 1068 shows a coherent energy-dependent behavior despite the multiple instruments, observational apertures and observational dates. The linear continuum polarization is the highest in the ultraviolet band where starlight emission is weak: the starlight fluxes of spiral galaxies are about three orders of magnitude lower at $0.1 \mu \mathrm{m}$ than at $1 \mu \mathrm{m}$ (Bolzonella et al. 2000, Siebenmorgen \& Krügel
2007). With increasing diluting fluxes from starlight, the continuum polarization of NGC 1068 decreases from $\sim 15 \%$ at $0.1-0.2 \mu \mathrm{m}$ to $\sim 1 \%$ at $0.8-0.9 \mu \mathrm{m}$. The polarized flux, despite being not as well sampled as the polarization degree due to the lack of reported flux measurements, clearly shows the turnover of host dominance. At ultraviolet wavelengths the polarized flux is constant while it decreases sharply in the optical, dipping at $\sim 1 \mu \mathrm{m}$. The dip in polarization at $\sim 1 \mu \mathrm{m}$ is due to the maximum of starlight contribution that almost cancel the observed polarization (Bolzonella et al. 2000). This polarization dip is also consistent with the transition waveband between the "Big Blue Bump" and the "infrared bump" detected in the spectral energy distribution of AGN. The Big Blue Bump is due to multi-color black body emission from the accretion disk and the infrared bump is attributed to thermal emission from dust (Sanders et al. 1989 Wilkes 2004). The inflection between the two bumps, related to dust sublimation at temperature $1500-2000 \mathrm{~K}$, occurs at $1-2 \mu \mathrm{m}$, similarly to the onset of the infrared polarized peak. As it can be seen in Fig. 2, both the polarization degree and the polarized flux are strongly increasing in the near-infrared band. Interestingly, it appears that the polarized flux spectrum rises much more sharply in the nearinfrared than the average type-1 SED that we have plotted. In this case, the orientation difference between NGC 1068 and the averaged SED of Prieto et al. (2010) certainly plays a role as polarization by polar scattering transitions in the infrared to polarization due absorption/emission by aligned grains (this will be developed in the next paragraph). Polar scattering provides a similar face-on view to what we see in type-1 AGNs, but in the case of dichroic polarization, we are seeing the transmitted (or emitted) light from the edge on torus dust distribution.

At longer wavelengths the host emission decreases and the infrared polarization shows a second maxima at wavelengths $2-3 \mu \mathrm{m}$. The absence of variation seen in the polarization position angle of light (that remains perpendicular to the axis of radio emission from the ultraviolet band to $\sim$ $3 \mu \mathrm{m}$ ) indicates that most of the polarization is due Thomson and Mie scattering in the polar region. However, at 4 $-5 \mu \mathrm{m}$, the polarization position angle switches from perpendicular to almost parallel. This effect was already highlighted and discussed by Bailey et al. (1988). The authors have shown that the angle rotation is not a consequence of the observed forbidden lines flux included in the passbands of the filters used, which would have the effect of pulling the position angle towards the larger forbidden line value, but it is in fact a real feature of the continuum po- 

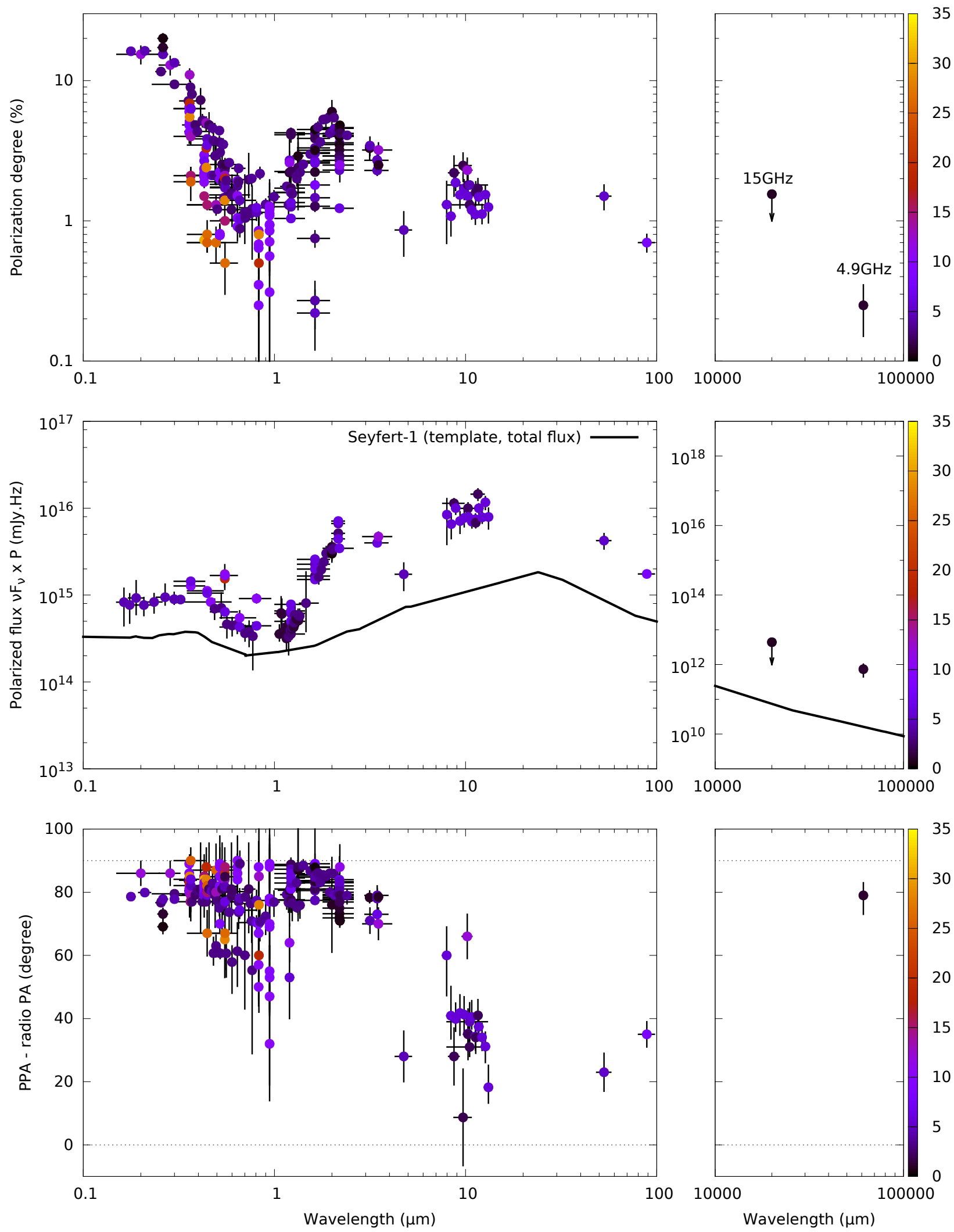

Figure 2. Broadband $0.1-100 \mu \mathrm{m}, 4.9 \mathrm{GHz}$ and $15 \mathrm{GHz}$ continuum polarization of NGC 1068 measured from various instruments and apertures (color-coded, in arcseconds). Instrumental and interstellar polarization have been accounted for but contribution from the host galaxy is still present. See text for details. Top: polarization degree, middle polarized flux $\left(\nu \mathrm{F}_{\nu}\right.$, in $\mathrm{mJy} . \mathrm{Hz}$, times $\left.P\right)$, bottom: polarization position angle (PPA) minus the parsec-scale radio position angle (PA). The Seyfert 1 total flux template presented in the polarized flux figure (middle) was shifted downward for better visibility. 
larization. While the polarization in the ultraviolet, optical and near-infrared bands is caused by polar scattering by electron and dust grains, the polarization at $\lambda \geqslant 4 \mu \mathrm{m}$ is most likely due to thermal emission from 100 pc dust grains aligned by large-scale magnetic fields (Efstathiou et al. 1997. Lopez-Rodriguez et al. 2015). Since most the dust is concentrated along the AGN equatorial plane, the polarization angle aligns with the radio axis and becomes parallel. The sharp decrease of polarized flux we observe at $4-5 \mu \mathrm{m}$ confirms the variation of the cross section of the scatterer. We are then able to trace the exact wavelength at which polar scattering becomes inefficient in NGC 1068.

In mid- and far-infrared bands, $10 \mu \mathrm{m}$ polarization observations at subarcsecond resolution showed that the core of NGC 1068 becomes consistent with zero measured polarization; the main contributor to polarization is the extended emission that comes from the polar material / GMC (giant molecular clouds) interactions in the Northern ionization cones (Lopez-Rodriguez et al. 2016). Such discovery highlights the importance of correlating near- and mid-infrared data to draw conclusions. The polarized flux follows the dust emission by aligned grains and strongly increases between 10 and $20 \mu \mathrm{m}$. The polarized flux appears to reach a maximum in the uncharted $20-40 \mu \mathrm{m}$ and far-infrared polarimetric measurements indicate that the polarized flux decreases in the $50-100 \mu \mathrm{m}$ waveband.

Finally, in the radio band, two Very Large Array (VLA) measurements of the core polarization of NGC 1068 have been published. An upper limit was estimated at $15 \mathrm{GHz}$ and the $4.9 \mathrm{GHz}$ apparent polarization of the central AGN component is at the limit of accuracy of the measurement technique. Nevertheless, the polarized flux follows the expected trend of the type- 1 total flux template with great consistency. The polarization degree is low, of the order of $0.25 \%$ at $4.9 \mathrm{GHz}$ and below $1.5 \%$ at $15 \mathrm{GHz}$. Such low polarization degrees may be attributed to dilution by large quantities of thermal electrons as evidenced by the intense optical emission lines (Kraemer et al. 1998). The polarization angle could only be measured at $4.9 \mathrm{GHz}$ and is essentially perpendicular to the source axis, similarly to the optical continuum polarization (e.g., Martin et al. 1983). We thus detect a second rotation of the polarization position angle from the farinfrared to the radio domain. This rotation points toward a mechanism that is parallel to the polar magnetic fields. Accounting for the low polarization degree due to dilution by thermal electrons and the polarization position angle that is perpendicular to the radio-axis, electron-scattered synchrotron emission appears to be the most plausible scenario (Gallimore et al. 2004, Krips et al. 2006). This is in agreement with the work of Krips et al. (2006) who investigated several emission mechanisms to explain the millimeter-toradio continuum emission in NGC 1068 . Indeed, the authors concluded that "the core fluxes indicate a turnover of the inverted cm- into a steep mm-spectrum at roughly $50 \mathrm{GHz}$ which is most likely caused by electron-scattered synchrotron emission".

In conclusion, it is truly remarkable to observe that the whole polarized SED of NGC 1068 is following the averaged total flux type-1 SED extracted from Prieto et al. (2010), proving that a type-1 core genuinely resides inside NGC 1068. We also note that the different emission and reprocessing mechanisms have a deep impact onto the po-
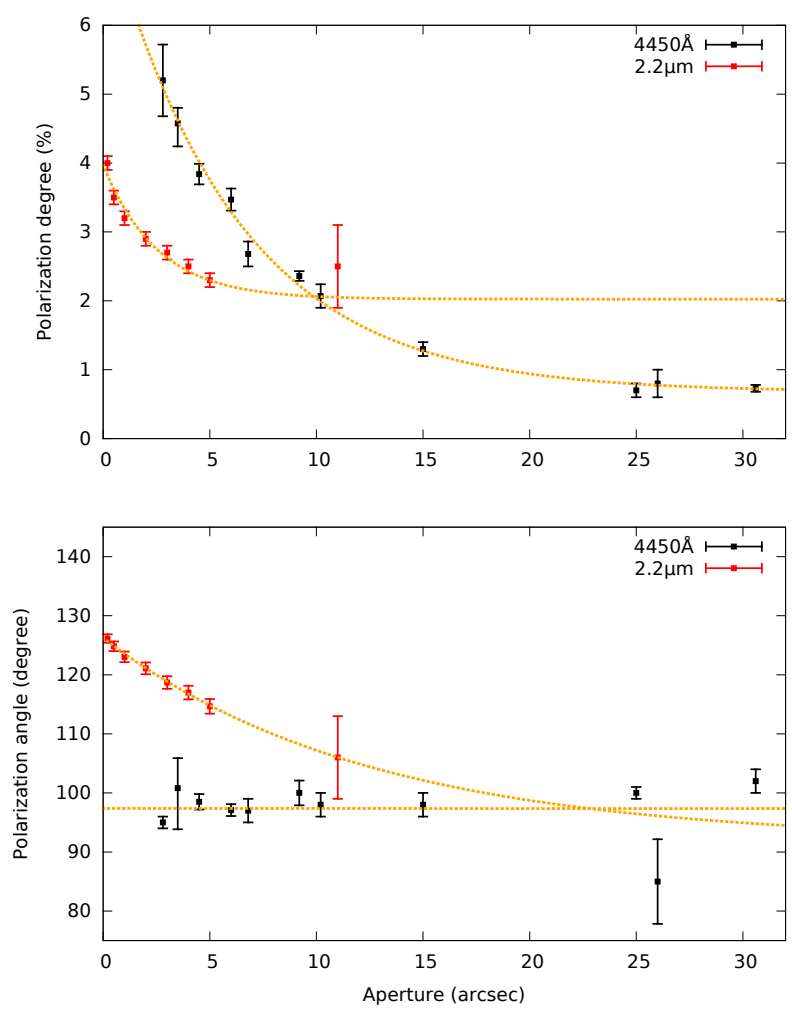

Figure 3. Aperture effect on the measured continuum polarization of NGC 1068 around $4450 \AA$ (in black) and around $2.2 \mu \mathrm{m}$ (in red). The first panel shows the variations of the polarization degree, the second panel is for the polarization position angle. Fits to the data are shown in orange dashed lines.

$$
\begin{array}{l|l}
\mathrm{P}_{4450}=0.68+6.99^{*} \exp \left(-0.16^{*} \mathrm{Ap}\right) & \mathrm{R}^{2}>0.99 \\
\mathrm{P}_{2.2 \mu \mathrm{m}}=2.02+1.95^{*} \exp (-0.39 * \mathrm{Ap}) & \mathrm{R}^{2}>0.97 \\
\hline \Psi_{4450 \AA}=-0.49 .10^{-4 *} \mathrm{Ap}+97.37 & \mathrm{R}^{2}<0.01 \\
\Psi_{2.2 \mu \mathrm{m}}=91.90+34.34^{*} \exp \left(-0.08^{*} \mathrm{Ap}\right) & \mathrm{R}^{2}>0.99
\end{array}
$$

Table 3. Fits to variation of polarization degree $P$ and polarization position angle $\Psi$ as a function of aperture $A p$ from Fig. 3 The coefficient of determination $\mathrm{R}^{2}$ is indicated in the second column.

larization position angle, allowing us to probe the physics inside the core of obscured AGN.

\subsection{Impact of aperture onto the observed polarization}

It is well known that the measured AGN polarization depends on the aperture used (see, e.g., Bailey et al. 1988). The better we isolate the compact nucleus from the host starlight and starburst activity, the higher the ultraviolet, optical and near-infrared polarization degre 5 . This effect is

5 Thanks to high angular-resolution polarimetric observations, Packham et al. (2007) and Lopez-Rodriguez et al. (2016) have shown that the $10 \mu \mathrm{m}$ polarization decreases with decreasing aperture. This is due to the complex combination of a) the extended emission from the wind-GMC interaction in the North- 
clearly visible in Fig. 2, where the color-code highlights the fact that polarimetric measurements with large apertures are always smaller than polarimetric observations at the same wavelength with a smaller observational aperture. Yet, since our catalog compiles all polarimetric data recorded for NGC 1068, we can investigate the impact of aperture onto the resulting polarization with better accuracy. We isolate two regions from Fig. 2 where the polarization degree is found to vary over several percentage points at a given wavelength: around $4450 \AA$ (B-band) and around $2.2 \mu \mathrm{m}$ (K-band). The polarization dependency on the aperture is shown in Fig. 3 (top: polarization degree, bottom: polarization angle). The aperture varies from 2.8" to 30.6 " in the blue band and from 0.2 " to 11 " in the infrared, which corresponds to $205-2250 \mathrm{pc}$ and $14-810 \mathrm{pc}$, respectively. We see that the observed polarization degree indeed increases gradually as we get closer to the active nucleus. Dilution by the host is relatively important for large apertures and the polarization degree exponentially increases when the observer reaches apertures lower than 15 ". We fitted the data point using exponential function that are summarized in Tab. 3 All fits have a coefficient of determination $\mathrm{R}^{2}$, that is the proportion of the variance in the dependent variable that is predictable from the independent variable, superior to 0.97. However, there is no evidence for a variation in the polarization position angle at $4450 \AA$ as aperture increases. Overall, we find a clear exponential dependence of the polarization properties with respect to the observational aperture. Our fits can then help to predict the expected polarization degree and angle at a given aperture for a given waveband. Additional polarimetric observations are needed in a large variety of wavebands to generalize our equations.

The observed polarization plateaus and the rotation of the polarization position angle indicate the presence of large scale polarization that can only be attributed to the host itself. Scattering of starlight by dust, molecules and electrons in the galactic medium is known to produce a low amount of optical polarization that depends on the orientation of the host plane (Scarrott et al. 1991; Simmons \& Audit 2000). For an edge-on galaxy, the expected large scale polarization lies between 0.8 and $1.8 \%$ (Simmons \& Audit 2000). The former value well corresponds to the plateau reached by the optical polarization curve in Fig. 2(top). This polarization degree is in agreement with the optical linear polarization maps of NGC 1068 obtained by Scarrott et al. (1991), who have shown that the polarization data from $r<10$ " traces the large-scale structure of the host. The spiral structure of the host, forming a roughly circularly symmetric pattern, almost certainly accounts for some of the decrease in polarization as the aperture size increases. At longer wavelength, near-infrared polarimetry of a normal spiral galaxy allowed Clemens et al. (2013) to show that the polarization fraction of late-type galaxies can reach up to $3 \%$, which also corresponds to the plateau of $2.2 \mu \mathrm{m}$ polarization we found. In conclusion, the diversity of apertures used to achieve polarimetric observations of NGC 1068 allowed us to confirm

ern ionization cones, b) the absorption polarization in the Southern cone, and c) the unpolarized core from self-absorbed dichroic emission from the torus.

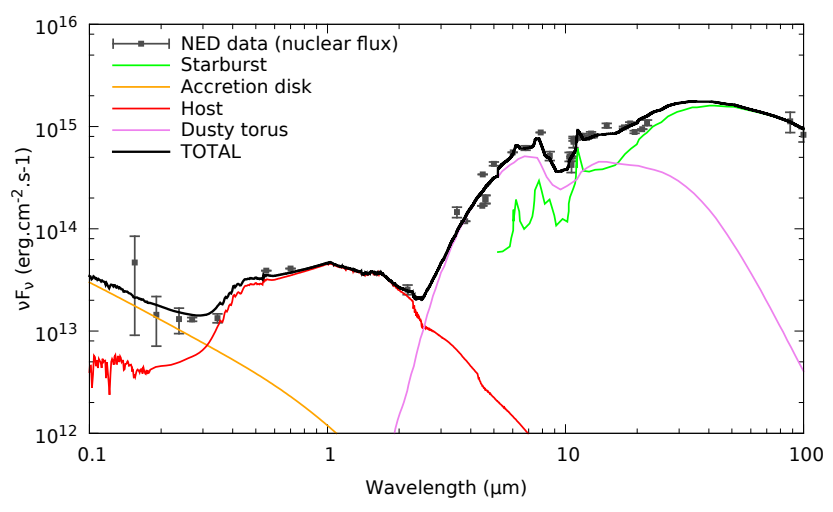

Figure 4. Observed nuclear fluxes of NGC 1068 (gray squares) extracted from the NASA/IPAC Extragalactic Database (NED). See text for details about the emissive components used to reproduce the broadband SED.

the amount of optical and near-infrared polarization from its host.

Additionally, according to Schmitt \& Kinney (1996) and Murayama \& Taniguchi (1997) the optical size of the extended narrow line region in NGC 1068 is about $900 \mathrm{pc}$ (12.25"), which roughly corresponds to the inflexion point of the optical polarization fit as a function of aperture in Fig. 3 Since most of the low-aperture optical continuum polarization we observe from NGC 1068 is due to scattering of disk photons inside the polar outflows, and since we have demonstrated that large aperture polarization is dominated by the host, we can safely confirm that the extension of the narrow line region probably stops before 1030 pc. This value is at a safe distance from the abrupt fall in the surface brightness profile of NGC 1068 observed by Sánchez-Portal et al. (2004) and that is situated around $2.2 \mathrm{kpc}$ from the center of the galaxy.

Consequently, despite the fact that the compiled continuum polarization spectrum of NGC 1068 presented in Fig. 2 suffers from the various apertures used, we know from Tab. 1 that only a few observations were done with apertures larger than the estimated extension of the scattering outflows. This indicates that most of the data have indeed measured the polarization originating mainly from the first hundreds of parsecs surrounding the AGN core and that the features we see in Fig. 2 are not artifacts. Even if all the different apertures do play a role, they only contribute to a lesser extent to the characteristic wavelength-dependent polarization profile.

\subsection{Reconstructing the spectral energy distribution}

Correcting the continuum polarization from diluting light is a major complication. While it is important to estimate the instrumental polarization and the contamination by interstellar polarization, it is crucial to remove the dilution by starlight in order to study the wavelength dependence of polarization (Miller \& Antonucci 1983). To do so, the aforementioned authors carried out observations of M32, a morphologically classified compact elliptical galaxy, in order to remove any unpolarized starlight that is dominating the continuum polarization of NGC 1068 longwards of $4000 \AA$. By 
minimizing the stellar absorption features in the residuals of the M32/NGC 1068 flux ratio spectra, Miller \& Antonucci (1983) derived the following starlight fractions in the observed AGN continuum flux: 0.42 at $3600 \AA, 0.63$ at $4200 \AA$, 0.78 at $4600 \AA$, and 0.82 at $5075 \AA$ (with $5 \%$ uncertainty). They corrected their linear polarization spectrum and found a wavelength-independent polarization of $16 \pm 2 \%$ in the $3500-5200 \AA$ waveband. However, to achieve so, highresolution $(<10 \AA)$ spectropolarimetry is necessary. Most of the published polarimetric measurements of NGC 1068 were achieved in narrow-band filters, which prevent us to use this method.

We thus opted for a similar procedure that rather focuses on the continuum flux of NGC 1068. We extracted from the NASA/IPAC Extragalactic Database all the nuclear observations of NGC 1068 and plotted them in Fig. 4 We focused on nuclear fluxes in order to better estimate the fraction of starlight in the observed continuum flux of NGC 1068. We reconstructed the $0.1-100 \mu \mathrm{m}$ spectral energy distribution (SED) of NGC 1068 using usual AGN components: the scattered light of a thermally-emitting multicolor accretion disk, a component reproducing the infrared reemission of the circumnuclear AGN torus, a template for the host galaxy, and a template for the starburst light contribution.

- To construct the accretion disk SED, we used the standard thin disk model (Shakura \& Sunyaev 1973). The accretion disk spans from 1 to 1000 gravitational radii and surrounds a $8.10^{6}$ solar mass black hole (Lodato \& Bertin 2003) accreting at 0.4 times the Eddington rate (Kumar 1999). The inclination of the disk was fixed to $85^{\circ}$ according to the numerical reconstruction of NGC 1068 achieved by Fischer et al. (2013, 2014). The question about the true inclination of the system remains opened (Marin et al. 2016) but we checked that varying those parameters only marginally influence the power-law shape of the scattered light of the disk SED observed in the ultraviolet-optical band. The variation are within the expected range of values (power-law index $\sim$ $1 / 3$ due to the superposition of black-bodies).

- Dust reemission by the obscuring equatorial torus is simulated using the model presented by Fritz et al. (2006) to fit the infrared SED of NGC 1068. The toroidal model has an aperture angle of $160^{\circ}$, an optical depth of 8 at $9.7 \mu \mathrm{m}$, and is characterized by multiple grain temperatures set by thermal equilibrium equations. The torus extends up to $16.4 \mathrm{pc}$, has an outer-to-inner radii ratio of 20 and its dust density distribution varies both in the radial and in the altitude coordinates. We note that Fritz et al. (2006) used a homogeneous distribution of dust to describe the torus; in reality ALMA has shown that the outer edge of the torus is likely to be clumpy García-Burillo et al. 2016, Gallimore et al. 2016). Its outer radius is of the order of $10 \mathrm{pc}$ but most of the obscuring material of the torus is concentrated around 5 pc, according to the clumpy torus models (García-Burillo et al. 2016). This results in a model that may overestimate the far-infrared/millimeter emission that is, in any case, extended.

- A template for the host galaxy has been extracted from Bruzual A. \& Charlot (1993) and was extended towards the ultraviolet and infrared bands by Bolzonella et al. (2000). It corresponds to an archetypal Sbc barred spiral galaxy,

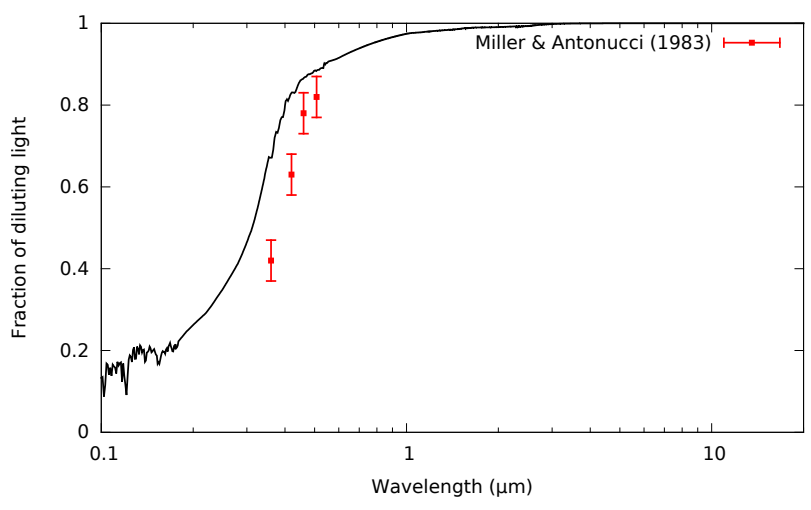

Figure 5. Derived fraction of diluting light (starlight plus dust emission components) in the observed continuum flux of NGC 1068. The previous estimation made by Miller \& Antonucci (1983) using a circular aperture of 2.8 " is shown in red.

which is consistent with the classification of the host of NGC 1068 (Balick \& Heckman 1985). The initial mass function by Miller \& Scalo (1979) was used with an upper mass limit for star formation of $125 \mathrm{M}_{\odot}$. The database used to compute this SED includes only solar metallicity SEDs but it appears to have a minimum impact onto the resulting template (Bolzonella et al. 2000).

- Finally, starburst activities are present in the central kilo-parsec of NGC 1068 (Lester et al. 1987, Romeo \& Fathi 2016), imprinting the $5-100 \mu \mathrm{m}$ infrared band with strong features (Thronson et al. 1989 . Le Floc'h et al. 2001). Following Fritz et al. (2006), we included a starburst component needed to reproduce the intensity of the polycyclic aromatic hydrocarbon features between 6 and $15 \mu \mathrm{m}$, as well as the depth of the silicate feature at $9.7 \mu \mathrm{m}$, and the width, intensity and peak wavelength of the infrared bump. To do so, we include the contribution of the infrared spectrum of the starburst galaxy NGC 7714 that correctly reproduces the colder component of dust emission (Fritz et al. 2006).

Our final SED is presented in black in Fig. 4 and it satisfactorily reproduces observations (in gray). This SED has been chosen to minimize the differences between the model and the observed fluxes but we remark slight degeneracies due to the data errors bars, particularly in the ultraviolet and blue bands. Nevertheless, the shape of the near and midinfrared spectrum is very well reproduced using a generic template for the host rather than using a specific galaxy observation such as M32. The transition between disk emission and torus emission happens at the expected wavelength and the host galaxy indeeds dominates the continuum flux of NGC 1068 longwards of $3000 \AA$.

\subsection{Polarization correction}

The overall agreement between the observed data points and our SED model allows us to derive the fraction of starlight plus dust emission components (dust-reprocessed accretion disk emission and starburst emission) in the observed continuum flux of NGC 1068. The flux ratio is shown in Fig. 5 , together with the ratios derived by (Miller \& Antonucci 1983) using the M32 template. Although the ratio are not the same (this being due to the two different approaches: spectropo- 


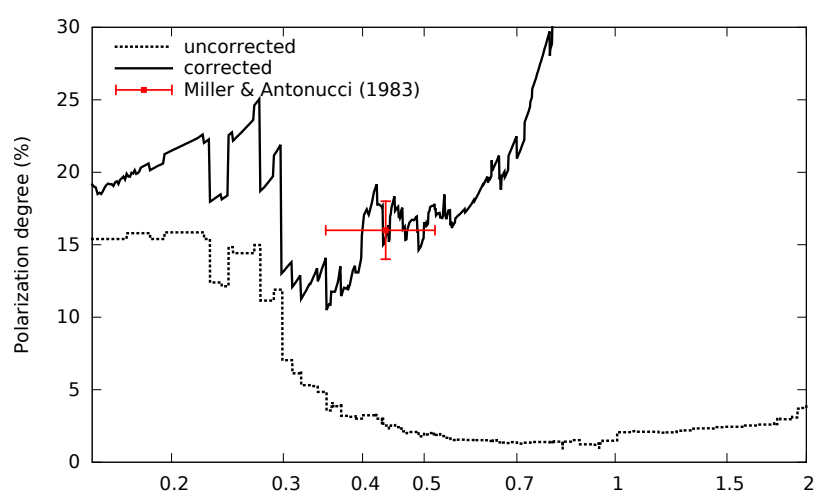

Figure 6. Corrected average continuum polarization of NGC 1068 using the fraction of diluting light (starlight plus dust emission components) in the observed continuum flux presented in Fig. 5The previous estimation made by Miller \& Antonucci (1983) is shown in red.

larimetric fitting versus SED fitting, the former being unfeasible in this paper), the shape of the wavelength-dependent ratios are distinctively similar. In our model, the host galaxy starlight starts at bluer wavelengths, hence the shift by almost $1000 \AA$. We tried to reproduce the exact same flux ratios estimated by (Miller \& Antonucci 1983) by changing the normalization of our SED components but the observed data points were no longer matched in the optical band. We stress that this is a logical outcome: first there is a difference in the apertures being used; second the two host galaxy SED used in Miller \& Antonucci (1983) and in our paper are not the same. In particular the choice of M32 may have had consequences on the spectropolarimetric analysis. M32 is an elliptical satellite galaxy of the M31 subgroup, together with NGC 205. A giant stream in the outer halo of M31, pointed in the direction of M32, was observed by Ibata et al. (2001), leading to potential tidal interaction between the galaxies (Choi et al. 2002). But, despite the morphological and chemical differences between the two templates, the most important investigation is to test whether our SED and the fraction of starlight plus dust emission components in the observed continuum flux of NGC 1068 can reproduce the expected polarization thresholds quoted by Miller \& Antonucci (1983) and Antonucci \& Miller (1985).

In Fig. 6, we present the averaged combined polarization spectrum of NGC 1068 taken from Fig. 2 (dashed line), together with the linear continuum polarization corrected for 1) the host starlight dilution and 2) the dust emission components, both the torus (i.e. reprocessed accretion disk emission) and the starburst dust emission, using the derived fraction of diluting light in the observed continuum flux of NGC 1068 (Fig. 5). We added the measure by Miller \& Antonucci (1983) in red and we cut the spectrum at $2 \mu \mathrm{m}$ since dust reemission is dominating at longer wavelengths. Our correction to the averaged compiled polarization spectrum of NGC 1068 reproduces the measurement by Miller \& Antonucci (1983) in the optical band. Our data indicates a median polarization of $17 \pm 3 \%$ in the $3000-7000 \AA$ band while Miller \& Antonucci (1983) estimated a continuum polarization of $16 \pm 2 \%$ in the $3500-5200 \AA$ waveband. Our method is subject to higher polarization fluctuations due to the large range of observational apertures used but gives reli- able estimation of the true scattered polarization of Seyfert2 AGN. The sharp features at $\sim 0.22 \mu \mathrm{m}$ and $\sim 0.3 \mu \mathrm{m}$ are directly caused by the lack of polarimetric measurements in several consecutive bins (as it can be seen in the uncorrected spectrum in dashed line that sharply decreases at those wavelengths). It also appears that the continuum polarization increases in the ultraviolet due to dust scattering at distances larger than 1" from the AGN core (Antonucci et al. 1994). This is in complete agreement with the discovery of Hönig et al. (2013) and Asmus et al. (2016), who have shown that optically thin dust in the polar outflows is responsible for much for the observed mid-infrared flux. The ultraviolet wavelength-dependent polarization signature we observe in Fig. 6] (for apertures greater than 1") is naturally explained by scattering of light by dust particles along the polar direction. In the near-infrared band the asymptotic behavior of the dilution-corrected polarization spectrum is a consequence of the fact that the accretion disk continuum decreases relative to the stellar continuum, as wavelength increases. Nevertheless, we demonstrated that it is possible to build a reliable combined spectrum of the optical linear continuum polarization of AGN from published data despite the diluting action of the host galaxy and dust emission components.

\subsection{Temporal evolution of the continuum polarization}

The last aspect of our study is to test whether the polarization of NGC 1068 has significantly evolved in time. We know that the observed polarization does not linearly depend on the amount of photons produced by the central engine. The chaotic light curve of AGN do not change the polarization state unless the powerful radiation field wipes out a fraction of the material in the vicinity of the black hole (see, e.g., Lawrence 1991, Jackson \& Browne 1990, Hill et al. 1996). Even in this case, the amount of material removed by the radiation field must be significant to alter the polarimetric signature of the AGN in a detectable fashion (Marin et al. 2016). The dynamical timescales for changing the geometric arrangement of matter or magnetic fields at (sub-)parsecscales around a $10^{8}$ solar masses black hole is of the order of 5 - 100 year (Hopkins et al. 2012). Only long-term monitoring of the polarization of a given AGN can probe such changes.

We present in Fig. 7 the time evolution of the continuum polarization of NGC 1068 in several wavebands from the optical to the infrared domain. A similar aperture, indicated in each plot, was chosen when compiling the published data in order not to include systematic effects. There can still be (for the smallest apertures) a small impact of the seeing but this is likely mitigated by the use of a range of apertures rather than a single aperture. As it can be seen, the polarization degree does not change by more than a fraction of a percentage point over several decades. If the error bars of the first and last polarimetric points in the $3800-4880 \AA$ and $1.8-2.6 \mu \mathrm{m}$ figures are real, then there is a tentative statistically significant increase of polarization with time but the polarization angle (not shown) remains constant. This could coincide with the variability of the near-infrared nuclear flux of NGC 1068 observed between 1976 and 1994 by Glass (1997). From his study, it was not clear whether this 

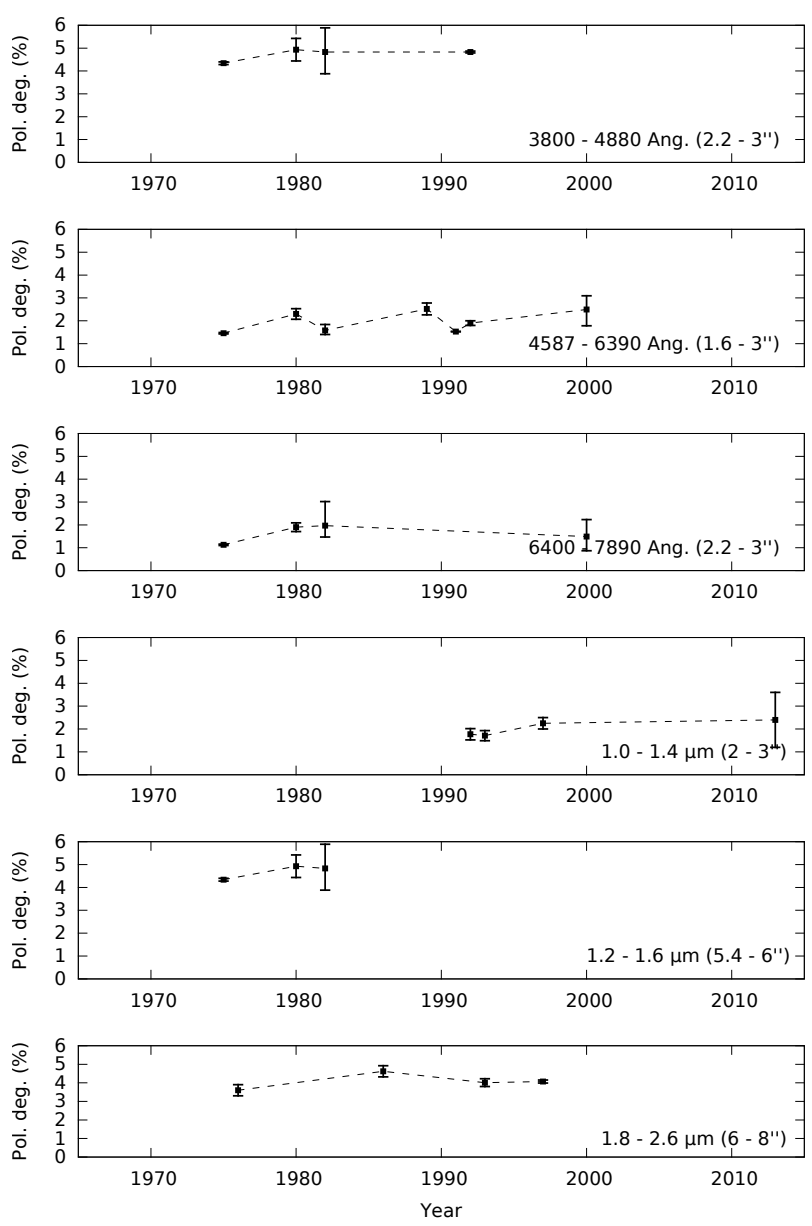

Figure 7. Time evolution of the polarization degree of NGC 1068 in several wavebands at a fixed aperture.

event has been the response of an extended dusty region to a single outburst in the central engine or the result of a continuous change in its ultraviolet output, but it was shown in Marin et al. (2016) and Marin (2017) that parsec-scale morphological changes in the geometry of the AGN would have varied the observed polarization degree by several percentage points and may be accompanied by a rotation of the polarization position angle. It is then safe to say that the global morphologic and magnetic geometry of NGC 1068 probably did not change since 1975 . The $1965-1975$ period could not be investigated due to the large apertures used at that time, but it is unlikely that a strong change happened in less than a decade. Overall, we have proven that NGC 1068 remained remarkably constant in terms of polarimetry over the past 50 years.

\section{MULTI-WAVELENGTH POLARIMETRY OF AGN: WHAT IS MISSING?}

Reconstructing the polarization spectrum of an AGN is a difficult task since polarimetric data are easily contaminated by starlight, Galactic dust, starburst light and interstellar polarization. However, we have shown that the polarimetric spectrum of NGC 1068, despite the diversity of instruments and apertures, has distinctive wavelength-dependent signatures that can be related to the AGN and host galaxy physics. Impact of the Big Blue bump and infrared bump, electron, Mie and dichroic scattering, synchrotron emission and radio dilution by thermal electron are all naturally highlighted in polarization. This is the reason why polarimetry remains one of the best methods to explore the geometry and physics of unresolvable/obscured cosmic sources. Nevertheless an important fraction of the polarized spectrum of NGC 1068 remains unexplored.

The X-ray band is a territory where no polarimetric information has ever been recorded in the case of AGN. Xray polarization is expected to arise from electron, dust and gas scattering, as well as magnetic processes (McNamara et al. 2009). Compton and inverse-Compton scattering are the dominant processes, similarly to Thomson and Mie scattering in the optical band, and the expected X-ray polarization can be evaluated (at first order) by looking at the visual polarization (Marin et al. 2016). In addition, since the $\mathrm{X}$-ray continuum is produced close to the central supermassive black hole, special and general relativistic effects are expected to impact the observed polarization properties of light (e.g. Connors \& Stark 1977; Pineault 1977, Connors et al. 1980). Due to strong gravity effects, the polarization direction rotates along the photon null geodesics as the polarization vector is parallelly transported, resulting in specific polarimetric features. By observing the X-ray polarization of compact objects, it becomes possible to characterize the mass of the black hole, its spin, the composition of the accretion disk and the geometry of the system (Dovčiak et al. 2004 Schnittman \& Krolik 2009, 2010 Marin \& Weisskopf 2017. Marin et al. 2018).

The far-ultraviolet band offers unique insight into the physics of AGN that is still little known, in particular by probing ultraviolet-emitting and absorbing material arising from accretion disks, synchrotron emission in jet-dominated AGN and large-scale outflows. Some key signatures of accretion disks can be revealed only in polarized light, and with higher contrast at ultraviolet than at longer wavelengths (Kishimoto et al. 2008). Specifically, ultraviolet polarimetry can provide geometrical, chemical and thermodynamical measurements of accretion disks at unprecedented resolutions. By probing the ubiquitous magnetic fields, which are expected to align non-spherical small dust grains on the scales of the accretion disk to the extended torus, a future ultraviolet polarimeter such as POLLUX on-board of LUVOIR (Bolcar et al. 2016; Bouret et al. 2018) will be able to reveal the mechanisms structuring the multi-scale AGN medium.

Far-infrared polarimetry remains an almost uncharted waveband despite the fact that observations do not suffer from seeing problem, allowing to deeply probe high extinction areas. Polarimetric measurements of ultra-luminous galaxies would allow one to address a variety of issues such as the nature of the emission that could be either nonthermal or originating from dust. By detecting solid state spectral features, far-infrared polarimetry should be able to discriminate between thermal and non-thermal emission in AGN, such as starburst galaxies (Bressan et al. 2002 Andreani et al. 2003). The large-scale polarimetric signature most likely originates from extended magnetic fields that align dust grains. The associated dichroic absorption and emission mechanisms provide information on the geometry 
of the magnetic fields, which is essential to better understand accretion processes, disk formation and mass outflows from stars to AGN (Hough \& Aitken 2003).

Polarimetric imaging in the (sub)millimeter offers the possibility of identifying magnetic field configurations at unprecedented scales. Using ALMA, we can resolve the torus outer regions and study the polarized dust emission by dichroic emission and absorption by aligned grains. In particular Aitken et al. (2002) have shown that sub-millimeter polarization can lead to strong constraints on the field configuration for a variety of torus models. Circular polarization, that is easily acquired at those wavelengths, could also enlighten us on the jet structure of AGN (Sazonov 1969). The POLAMI program (Agudo et al. 2018a b | Thum et al. 2018 ) is using the fact that Faraday conversion can convert linear polarization to circular polarization to probe the magnetized plasma of jet in a large sample of radio-loud AGN.

Finally AGN radio polarization measurements are possible as synchrotron emission is naturally highly polarized when magnetic field lines are ordered (Westfold 1959). However, in the case of radio-quiet AGN polarization, the $\mathrm{GHz}$ and $\mathrm{MHz}$ bands remain largely unexplored as Seyfert galaxies are generally reported to be unpolarized in the radio band, even in the optically thin regions (Antonucci 1993). Yet those measurements have been achieved almost four decades ago and NGC 1068 appears to be (weakly) polarized at $4.9 \mathrm{GHz}$. New observations with modern radio telescopes are necessary to explore with better sensitivity the emission and reprocessing mechanisms in the radio band. In particular the turnover frequency between dust polarized emission and synchrotron polarization could be detected by discovering the wavelength at which the polarization position angle rotates.

\section{SUMMARY AND CONCLUSIONS}

We have gathered all the published polarimetric information on NGC 1068, the most observed Seyfert galaxy in terms of polarimetry. We compiled the broadband $0.1-$ $100 \mu \mathrm{m}, 4.9 \mathrm{GHz}$ and $15 \mathrm{GHz}$ continuum polarization of this archetypal type-2 AGN using data from more than 50 years of observations, a premiere in the field. By doing so, we were able to detect all the expected transition regions in the polarized SED of NGC 1068: the Big Blue Bump $(0.1-0.7 \mu \mathrm{m})$, the peak of starlight contribution $(\sim 1 \mu \mathrm{m})$, the infrared bump $(1-4 \mu \mathrm{m})$, and the transition between electron scattering and polarized dust reemission $(4-5 \mu \mathrm{m})$. Additional radio measurements also point toward another polarization mechanism linked with synchrotron emission, scattering and dilution by thermal electrons, highlighted by a rotation of the polarization position angle between the farinfrared and the radio bands. Despite the large variety of apertures and instruments used, the wavelength-dependent behavior of the polarization clearly revealed the wavebands where processes are switching. This work allowed us to reconstruct the broadband polarized SED of NGC 1068 which strongly resembles to a typical type-1 SED seen in total flux. We analyzed the aperture effect on the measured continuum polarization of NGC 1068 and found a large scale polarization component that can only be attributed to the host itself. This constant value for high apertures corresponds to the measured polarization degree of regular spiral galaxies, confirming previous observational results (Scarrott et al. 1991 Simmons \& Audit 2000). For apertures lower than 15", the degree of polarization exponentially increases since the aperture gets smaller than the scattering region size, highlighting the presence of the narrow line region.

In order to carefully remove the contribution of starlight to the continuum polarization of our compiled spectrum, we reconstructed the global SED of NGC 1068 using nuclear fluxes from NED and a set of emissive components. By doing so, we were able to derive the fraction of starlight in the observed continuum flux of NGC 1068. We corrected our $0.15-2 \mu \mathrm{m}$ continuum polarization spectrum and found a wavelength-independent polarization level in the optical band, such as expected from theory and past studies. We also highlighted the impact of dust scattering in the ultraviolet and near-infrared bands for larger apertures (electron scattering is dominating from the ultraviolet to the nearinfrared at arc-second scale apertures). Finally, we checked whether the observed polarimetric signal of NGC 1068 varied through time. We demonstrated that this particular AGN did not undergo a major morphological or magnetic change over the past decades since its polarimetric signal remained constant (at similar apertures and in the same waveband).

In conclusion, we have created the very first broadband compiled polarization spectrum of an AGN exploiting more than 50 years of data. The results are in strong agreements with the Unified Scheme of AGN despite the scarcity of data at several wavebands. Large class $(30 \mathrm{~m})$ telescopes equipped with polarimeters are needed to pursue the study at deeper levels, both in spectroscopic and imaging modes. Despite being restricted to $0.1-100 \mu \mathrm{m}$, plus two points in the centimeter band, the work achieved in this paper can now be compared to the results of Monte Carlo radiative transfer simulations in order to test different geometries of the reprocessing and emitting media (e.g., Goosmann \& Gaskell 2007 Marin et al. 2012, 2015, Rojas Lobos et al. 2017. Grosset et al. 2017; Marin 2018). This is an important step as, so far, any model-to-data comparison was only achieved in narrow bands. We also highlight the fact that our study could be expanded by many orders of magnitude by looking at the radio, millimeter, far-infrared, far-ultraviolet and X-ray polarization of AGN using ALMA, HAWK+, or the forthcoming satellites IXPE (Weisskopf et al.|2016), eXTP (Zhang et al. 2016) and LUVOIR (Bolcar et al. 2016, Bouret et al. 2018).

\section{ACKNOWLEDGMENTS}

This paper is a tribute to all the observers who dedicated their career to better understand AGN using polarimetry. I would like to thank (in alphabetical order) Beatriz Agís González, Robert Antonucci, Lucas Grosset, Damien Hutsemékers, Enrique Lopez Rodriguez, Makoto Kishimoto, Chris Packham, Dominique Sluse, Yelena Stein and Bernd Vollmer for their numerous comments and suggestions that greatly improved this paper. Georges Roudnitski, from the Astronomicheskii Tsirkulyar journal, was of a great help to retrieve and translate old Russian papers. I also acknowledge the anonymous referee who helped to clarify and im- 
prove this paper. Finally, the author would like to thank the Centre national d'études spatiales (CNES) who funded this project through to the post-doctoral grant "Probing the geometry and physics of active galactic nuclei with ultraviolet and X-ray polarized radiative transfer".

\section{REFERENCES}

Agudo, I. 2016, PoS, 72

Agudo, I., Thum, C., Ramakrishnan, V., et al. 2018, MNRAS, 473, 1850

Agudo, I., Thum, C., Molina, S. N., et al. 2018, MNRAS, 474,1427

Aitken, D. K., Briggs, G., Bailey, J. A., Roche, P. F., \& Hough, J. H. 1984, Nature, 310, 660

Aitken, D. K., Efstathiou, A., McCall, A., \& Hough, J. H. 2002, MNRAS, 329, 647

Alexander, D. M., Young, S., \& Hough, J. H. 1999, MNRAS, 304, L1

Andreani, P., Cristiani, S., Grazian, A., La Franca, F., \& Goldschmidt, P. 2003, AJ, 125, 444

Angel, J. R. P., Stockman, H. S., Woolf, N. J., Beaver, E. A., \& Martin, P. G. 1976, ApJL, 206, L5

Antonucci, R. R. J. 1982, Nature, 299, 605

Antonucci, R. R. J. 1984, ApJ, 278, 499

Antonucci, R. R. J., \& Miller, J. S. 1985, ApJ, 297, 621

Antonucci, R. 1993, ARA\&A, 31, 473

Antonucci, R., Hurt, T., \& Miller, J. 1994, ApJ, 430, 210

Asmus, D., Hönig, S. F., \& Gandhi, P. 2016, ApJ, 822, 109

Bailey, J., Axon, D. J., Hough, J. H., et al. 1988, MNRAS, 234, 899

Balick, B., \& Heckman, T. 1985, AJ, 90, 197

Bauer, F. E., Arévalo, P., Walton, D. J., et al. 2015, ApJ, 812,116

Barthel, P. D. 1989, ApJ, 336, 606

Blaes, O., \& Agol, E. 1996, ApJL, 469, L41

Blaes, O., \& Agol, E. 1997, IAU Colloq. 163: Accretion

Phenomena and Related Outflows, 121, 610

Bolcar, M. R., Feinberg, L., France, K., et al. 2016, SPIE, 9904, 99040J

Bouret, J.-C., Neiner, C., Lopez Ariste, A., et al. 2018, American Astronomical Society Meeting Abstracts \#231, 231, \#419.01

Bressan, A., Silva, L., \& Granato, G. L. 2002, A\&A, 392, 377

Bruzual A., G., \& Charlot, S. 1993, ApJ, 405, 538

Bolzonella, M., Miralles, J.-M., \& Pelló, R. 2000, A\&A, 363,476

Capetti, A., Axon, D. J., Macchetto, F., Sparks, W. B., \& Boksenberg, A. 1995, ApJ, 446, 155

Capetti, A., Macchetto, F., Axon, D. J., Sparks, W. B., \& Boksenberg, A. 1995, ApJL, 452, L87

Choi, P. I., Guhathakurta, P., \& Johnston, K. V. 2002, AJ, 124,310

Clemens, D. P., Pavel, M. D., \& Cashman, L. R. 2013, AJ, 145, 74

Code, A. D., Meade, M. R., Anderson, C. M., et al. 1993, ApJL, 403, L63

Connors, P. A., \& Stark, R. F. 1977, Nature, 269, 128

Connors, P. A., Stark, R. F., \& Piran, T. 1980, ApJ, 235, 224
Dibai, E. A., \& Shakhovskoi, N. M. 1966, Astronomicheskij Tsirkulyar, 375 ,

Dombrovskii, V. A., \& Gagen-Torn, V. A. 1968, Astrophysics, 4, 163

Donahue, M., Connor, T., Fogarty, K., et al. 2015, ApJ, 805, 177

Dovčiak, M., Karas, V., \& Matt, G. 2004, MNRAS, 355, 1005

Dovčiak, M., Muleri, F., Goosmann, R. W., Karas, V., \& Matt, G. 2011, ApJ, 731, 75

Dyck, H. M., \& Jones, T. L. 1976, BAAS, 8, 568

Elvius, A., \& Hall, J. S. 1965, AJ, 70, 138

Elvius, A. 1978, A\&A, 65, 233

Efstathiou, A., McCall, A., \& Hough, J. H. 1997, MNRAS, 285, 102

Fischer, T. C., Crenshaw, D. M., Kraemer, S. B., \& Schmitt, H. R. 2013, ApJS, 209, 1

Fischer, T. C., Crenshaw, D. M., Kraemer, S. B., Schmitt, H. R., \& Turner, T. J. 2014, ApJ, 785, 25

Le Floc'h, E., Mirabel, I. F., Laurent, O., et al. 2001, A\&A, 367,487

Fritz, J., Franceschini, A., \& Hatziminaoglou, E. 2006, MNRAS, 366, 767

Gallimore, J. F., Baum, S. A., \& O'Dea, C. P. 2004, ApJ, 613,794

Gallimore, J. F., Elitzur, M., Maiolino, R., et al. 2016, ApJL, 829, L7

García-Burillo, S., Combes, F., Ramos Almeida, C., et al. 2016, ApJL, 823, L12

Gehrels, T. 1972, ApJL, 173, L23

Glass, I. S. 1997, Ap\&SS, 248, 191

Goosmann, R. W., \& Gaskell, C. M. 2007, A\&A, 465, 129

Gratadour, D., Rouan, D., Grosset, L., Boccaletti, A., \& Clénet, Y. 2015, A\&A, 581, L8

Grosset, L., Rouan, D., Gratadour, D., et al. 2017, arXiv:1712.02192

Hill, G. J., Goodrich, R. W., \& Depoy, D. L. 1996, ApJ, 462, 163

Hönig, S. F., Prieto, M. A., \& Beckert, T. 2008, A\&A, 485, 33

Hönig, S. F., Kishimoto, M., Tristram, K. R. W., et al. 2013, ApJ, 771, 87

Hopkins, P. F., Hayward, C. C., Narayanan, D., \& Hernquist, L. 2012, MNRAS, 420, 320

Hough, J. H., \& Aitken, D. K. 2003, Journal of Quantitative Spectroscopy \& Radiative Transfer, 79, 733

Hutsemékers, D., \& Lamy, H. 2000, A\&A, 358, 835

Ibata, R., Irwin, M., Lewis, G., Ferguson, A. M. N., \& Tanvir, N. 2001, Nature, 412, 49

Imanishi, M., Nakanishi, K., Izumi, T., \& Wada, K. 2018, ApJL, 853, L25

Jackson, N., \& Browne, I. W. A. 1990, Nature, 343, 43

Khachikian, E. Y., \& Weedman, D. W. 1974, ApJ, 192, 581

Kishimoto, M. 1999, ApJ, 518, 676

Kishimoto, M., Antonucci, R., Blaes, O., et al. 2008, Nature, 454, 492

Knacke, R. F., \& Capps, R. W. 1974, ApJL, 192, L19

Kraemer, S. B., Ruiz, J. R., \& Crenshaw, D. M. 1998, ApJ, 508, 232

Krips, M., Eckart, A., Neri, R., et al. 2006, A\&A, 446, 113

Kruszewski, A. K. 1968, AJ, 73, 852

Kruszewski, A. 1971, Acta Astronomica, 21, 311 
Kumar, P. 1999, ApJ, 519, 599

Landstreet, J. D., \& Angel, J. R. P. 1972, ApJL, 174, L127

Lawrence, A. 1991, MNRAS, 252, 586

Lebofsky, M. J., Kemp, J. C., \& Rieke, G. H. 1978, ApJ, 222, 95

Lester, D. F., Joy, M., Harvey, P. M., Ellis, H. B., Jr., \& Parmar, P. S. 1987, ApJ, 321, 755

Liu, J., Liu, Y., Li, X., et al. 2016, MNRAS, 459, L100

Lodato, G., \& Bertin, G. 2003, A\&A, 398, 517

Lopez-Rodriguez, E., Packham, C., Jones, T. J., et al. 2015, MNRAS, 452, 1902

Lopez-Rodriguez, E., Packham, C., Roche, P. F., et al. 2016, MNRAS, 458, 3851

Low, F. J., \& Rieke, G. H. 1971, Nature, 233, 256

Lumsden, S. L., Moore, T. J. T., Smith, C., et al. 1999, MNRAS, 303, 209

Marin, F., Goosmann, R. W., Gaskell, C. M., Porquet, D., \& Dovčiak, M. 2012, A\&A, 548, A121

Marin, F., Goosmann, R. W., \& Gaskell, C. M. 2015, A\&A, 577, A66

Marin, F., Goosmann, R. W., \& Petrucci, P.-O. 2016, A\&A, 591, A23

Marin, F., Rohatgi, A., \& Charlot, S. 2017, SF2A-2017: Proceedings of the Annual meeting of the French Society of Astronomy and Astrophysics, 113

Marin, F., \& Weisskopf, M. C. 2017, SF2A-2017: Proceedings of the Annual meeting of the French Society of Astronomy and Astrophysics, 173

Marin, F. 2017, A\&A, 607, A40

Marin, F., Dovčiak, M., Muleri, F., Kislat, F. F., \& Krawczynski, H. S. 2018, MNRAS, 473, 1286

Marin, F. 2018, A\&A, arXiv:1805.09098

Marinucci, A., Bianchi, S., Matt, G., et al. 2016, MNRAS, 456, L94

Martin, P. G., Thompson, I. B., Maza, J., \& Angel, J. R. P. 1983, ApJ, 266, 470

Mason, R. E., Wright, G. S., Adamson, A., \& Pendleton, Y. 2007, ApJ, 656, 798

McLean, I. S., Aspin, C., Heathcote, S. R., \& McCaughrean, M. J. 1983, Nature, 304, 609

McNamara, A. L., Kuncic, Z., \& Wu, K. 2009, MNRAS, 395, 1507

Miller, J. S., \& Antonucci, R. R. J. 1983, ApJL, 271, L7

Miller, G. E., \& Scalo, J. M. 1979, ApJS, 41, 513

Moran, E. C., Barth, A. J., Kay, L. E., \& Filippenko, A. V. 2000, ApJL, 540, L73

Murayama, T., \& Taniguchi, Y. 1997, PASJ, 49, L13

Muxlow, T. W. B., Pedlar, A., Holloway, A. J., Gallimore, J. F., \& Antonucci, R. R. J. 1996, MNRAS, 278, 854

Nikulin, N. S., Kuvshinov, V. M., \& Severny, A. B. 1971, ApJL, 170, L53

Packham, C., Young, S., Hough, J. H., Axon, D. J., \& Bailey, J. A. 1997, MNRAS, 288, 375

Packham, C., Young, S., Fisher, S., et al. 2007, ApJL, 661, L29

Prieto, M. A., Reunanen, J., Tristram, K. R. W., et al. 2010, MNRAS, 402, 724

Prunet, S., Sethi, S. K., Bouchet, F. R., \& MivilleDeschenes, M.-A. 1998, A\&A, 339, 187

Pineault, S. 1977, MNRAS, 179, 691

Raban, D., Jaffe, W., Röttgering, H., Meisenheimer, K., \&

Tristram, K. R. W. 2009, MNRAS, 394, 1325
Ramos Almeida, C., Martínez González, M. J., Asensio Ramos, A., et al. 2016, MNRAS, 461, 1387

Rojas Lobos, P. A., Goosmann, R. W., Marin, F., \& Savić, D. 2017, arXiv:1712.01147

Romeo, A. B., \& Fathi, K. 2016, MNRAS, 460, 2360

Rybicki, G. B., \& Lightman, A. P. 1986, Radiative Processes in Astrophysics, by George B. Rybicki, Alan P. Lightman, pp. 400. ISBN 0-471-82759-2. Wiley-VCH , June 1986., 400

Sánchez-Portal, M., Díaz, Á. I., Terlevich, E., \& Terlevich, R. 2004, MNRAS, 350, 1087

Sanders, D. B., Phinney, E. S., Neugebauer, G., Soifer, B. T., \& Matthews, K. 1989, ApJ, 347, 29

Sargsyan, L. A., \& Weedman, D. W. 2009, ApJ, 701, 1398

Sazonov, V. N. 1969, SvA, 13, 396

Scarrott, S. M., Rolph, C. D., Wolstencroft, R. W., \& Tadhunter, C. N. 1991, MNRAS, 249, 16P

Schartmann, M., Meisenheimer, K., Klahr, H., et al. 2009, MNRAS, 393, 759

Schartmann, M., Burkert, A., Krause, M., et al. 2010, MNRAS, 403, 1801

Schmitt, H. R., \& Kinney, A. L. 1996, ApJ, 463, 498

Schnittman, J. D., \& Krolik, J. H. 2009, ApJ, 701, 1175

Schnittman, J. D., \& Krolik, J. H. 2010, ApJ, 712, 908

Shakura, N. I., \& Sunyaev, R. A. 1973, A\&A, 24, 337

Siebenmorgen, R., \& Krügel, E. 2007, A\&A, 461, 445

Simmons, J. F. L., \& Audit, E. 2000, MNRAS, 319, 497

Smith, J. E., Young, S., Robinson, A., et al. 2002, MNRAS, 335,773

Simpson, J. P., Colgan, S. W. J., Erickson, E. F., et al. 2002, ApJ, 574, 95

Thronson, H. A., Jr., Hereld, M., Majewski, S., et al. 1989, ApJ, 343, 158

Thum, C., Agudo, I., Molina, S. N., et al. 2018, MNRAS, 473,2506

Tran, H. D. 1995, ApJ, 440, 565

Tran, H. D. 2003, ApJ, 583, 632

Urry, C. M., \& Padovani, P. 1995, PASP, 107, 803

Visvanathan, N., \& Oke, J. B. 1968, ApJL, 152, L165

Vollmer, B., Beckert, T., \& Davies, R. I. 2008, A\&A, 491, 441

Walker, M. F. 1964, AJ, 69, 744

Watanabe, M., Nagata, T., Sato, S., Nakaya, H., \& Hough, J. H. 2003, ApJ, 591, 714

Weisskopf, M. C., Ramsey, B., O'Dell, S., et al. 2016, SPIE, 9905, 990517

Westfold, K. C. 1959, ApJ, 130, 241

Wilkes, B. 2004, AGN Physics with the Sloan Digital Sky Survey, 311, 37

Wilson, A. S., \& Ulvestad, J. S. 1982, ApJ, 263, 576

Wilson, A. S., \& Ulvestad, J. S. 1983, ApJ, 275, 8

Wilson, A. S., \& Elvis, M. 1997, Ap\&SS, 248, 141

Young, S., Hough, J. H., Axon, D. J., Bailey, J. A., \& Ward, M. J. 1995, MNRAS, 272, 513

Young, S. 2000, MNRAS, 312, 567

Zhang, S. N., Feroci, M., Santangelo, A., et al. 2016, SPIE, 9905, 99051Q 